\title{
AN AGENT-BASED SIMULATION OF A JIT MATERIAL HANDLING SYSTEM
}

\author{
Qi Hao and Weiming Shen \\ Integrated Manufacturing Technologies Institute \\ National Research Council, Canada \\ 800 Collip Circle, London, Ontario N6G 4X8, Canada \\ [qi.hao;weiming.shen]@nrc.gc.ca
}

\begin{abstract}
Material handling is a loose loop in mast assembly plants. Just-in-time (JIT) is a management philosophy that strives to eliminate sources of manufacturing waste by producing the right part in the right place at the right time. We propose to apply JIT principles to material handling in assembly plants. Material Kanbans are introduced as an effective means to control and balance the physical materialpart flow in the plant floor. An agent-based simulation prototype is implemented using AnyLogic ${ }^{T M}$. The flexibility of the agent-based approach facilitates the simulation of various "what-if" scenarios inchding different layout designs, objective parameters and dynamic situations in the plant floor.
\end{abstract}

\section{INTRODUCTION}

Material handling is a loose loop that is generally neglected in most production plants. From our observation, even in a well designed assembly line, in condition that the whole line is optimized in its layout, processes, buffering, scheduling, and operations, material handling is still laid outside of the scope of control. Managers spend their precious time hunting everywhere for missing parts and arranging their deliveries. They are unaware of material handling/delivery schedules and the related resource information (amount and utilization of resources, such as forklifts and drivers). As a result, material handling becomes the major barrier that results in low efficiency, production breakdowns, and low quality of a production system.

Just-in-time (JIT) is a management philosophy that could improve profits and return on investment by reducing inventory levels, reducing variability, improving product quality, reducing production and delivery lead times, and reducing other costs (such as those associated with machine setup and equipment breakdown). The pull mechanism, especially introduced by the Kanban control of JIT manufacturing, enables an optimized production process that benefits from the cutting down of production resources. For a plant that already operates under a pull mechanism, material handling should also employ a pull mechanism rather than a MRP-based push mechanism. 
This paper intends to propose a pull material handling system based on principles in JIT manufacturing. In such a system, materials transportation in the plant floor is considered as individual tasks. Material Kanban (M-Kanban) is introduced as a carrier of delivery tasks which is an effective means to control and balance the physical material handling flow in the plant floor. The main principle behind is that a task generated by a production station (cell) requires the occupation of an M-Kanban to be delivered.

Another technology used in this material handling system is agent. Agent technology is evolved from the research domain of Distributed Artificial Intelligence in 1990s. From its emergence, agent technology is widely recognized as a promising paradigm for the next generation of design and manufacturing systems (Shen et al., 2001). In the JIT material handling simulation system, multiple agents are implemented to facilitate a collaborative problem solving environment. For example, each transportation vehicle is encapsulated as an agent so that it is manageable on its own parameters and behaviors, such as velocity, local schedule, and the associated scheduling, routing and conflict resolving rules. The driver of a transportation vehicle can deactivate a vehicle from the system to take personal activities or when the vehicle malfunctions and needs a repair. Moreover, the allocation of transportation task is accomplished though the negotiation of a Kanban scheduling agent and a number of vehicle agents. With such capacities, the system is able to simulate very dynamic situations and get more accurate information of transportation resources in general.

The rest of this paper is organized as follows: Section 2 reviews the background knowledge and literature of this study; Section 3 identifies a sample JIT material handling problem and describes the corresponding requirements; Section 4 proposes an agent-based architecture of the JIT material handling system and discusses two major design aspects: production simulation and material handling simulation; finally, Section 6 draws our conclusions.

\section{A TECHNOLOGY REVIEW}

There are two classifications of production control systems, namely push and pull. Material requirement planning (MRP) systems and Kanban control systems are the two most popular implementations of the push and pull strategies respectively. In a push production, in order to buffer inaccurate forecasts, inaccurate lead times, inaccurate inventory records, variable production schedules or questionable bill of materials (BOMs), MRP generally incorporates safety lead times and safe stocks. However, in practice, MRP may result in a serious problem of excessive inventories (Shirk, 1998; Hopp and Spearman, 1996). Stock levels and lead times are amplified down throughout the supply chain, from the final distributor down to each hierarchy of suppliers.

In contrast, using a pull strategy, a JIT system uses underutilized capacity instead of buffer inventories to hedge against problems that may arise. Production is initiated in response to real customer orders and the removal of items from the final distributor buffers triggers production upstream to replenish exhausted inventories layer by layer. Krishnamurthy et al. (2004) quantitatively compares the performance of MRP and Kanban for a multi-stage, multi-product manufacturing system. They 
reached the conclusion that pull strategies are handicapped for manufacturing facilities that produce a number of different products with distinct demands and/or processing requirements, as well as for facilities that make highly engineered products in small batches (even one-of-a-kind) for their customers.

The ideal industries that JIT production applies include automobile because it is where the JIT concept originated. The automotive industry is characterized by low product variety, and high-volume production. In an automotive assembly line, although there are some sub-lines using push strategies (sometime it is called hybrid production), such as the body shop, paint shop, and engine line, however, once cars are lining up to be processed on the main assembly line, the production is under control of a pure pull mechanism. Buffers are set at offline sites of sub-lines to tickle uncertainties and better serve the optimized production rate of the main assembly line.

The concepts of JIT and Kanban are never new. JIT were firstly developed by Toyota in the 1950's and adopted in the United States in the 1980's. Lean manufacturing and lean enterprise, proliferating in western countries, are also evolved based on JIT principles. Many small and medium sized businesses have embraced these concepts along with some of the major corporations such as, Mercedes/Benz, Pratt \& Whitney, Porsche and General Electric to name a few. Womack and Jones (1996) provide a thoughtful expansion upon their value-based business system based on the Toyota model. Along the way they update their action plan in light of new research and the increasing globalization of manufacturing, and they revisit some of their key case studies from the automotive, aerospace, and other manufacturing industries.

Many analytical, mathematical or experimental models are proposed to address the Kanban based operational planning and control issues (Berkley, 1992; Uzsoy and Martin-Vega, 1990). Simulation has been by far the methodology of choice in the majority of studies reported in the literature (Gupta and Al-Turki, 1998). Theoretically, the number of Kanbans and allocation of Kanbans in a system significantly affects the performance of a pull system. Instead of optimization of these Kanban arrangement which leads to a fixed number of Kanbans, Martins and Lewandrowski (1999) proposed a mathematical buffer stocks dimensioning approach using a dynamic kanban strategy. Gupta and Al-Turki (1998) compared the performance of a traditional kanban system (TKS) and a flexible kanban system (FKS). Through the simulation of two simple JIT models, they proved that FKS outperforms TKS under real-time manufacturing environments, such as sudden breakdown of a material handling system.

From application point of view, the researches of pull technologies could be classified in three categories: 1) production control; 2) inventory management; and 3) supply chain management (Kusiak, 2000). Material handling is a topic being previously researched in the literature (Gupta and Al-Turki, 1998; Askin, 1999; Venkataramanaiah et al., 2001), however, they all deal with the automatic material (specifically, the Work-In-Process, which belongs to the production line itself) transfer problem between production cells. The material we emphasize here does not refer to the WIP going through the assembly line, rather, it is the supply flow of material or parts subordinating to the main production line. None of known literature touched the topic of material handling from this aspect. 
Moreover, in most plants, material inventories are only virtually under control of either a MRP II system or a Kanban system at the enterprise level, but not physically at the dynamic plant floor. Missing parts, wrong part delivered, parts not at right place at right time are common occurrence in almost all mainstream production plants, including GM, Ford, and Sterling Truck. Material handling is a frustrating problem faced by production managers. Production managers are feeling nervous everyday and are blamed for lack of ability to control the manufacturing process. As a result, analysis of material handling and dynamic simulation will be of great help to industries.

\section{MATERIAL HANDLING SPECIFICATION OF A SIMPLIFIED ASSEMBLY LINE}

Simulation of material handling in a pull production setting is the primary purpose of this research. A Kanban-based material handling will be investigated to make it in line with the pull production line. For the convenience of a common understanding, a sample scenario is chosen as the background problem, as shown in Figure 1.

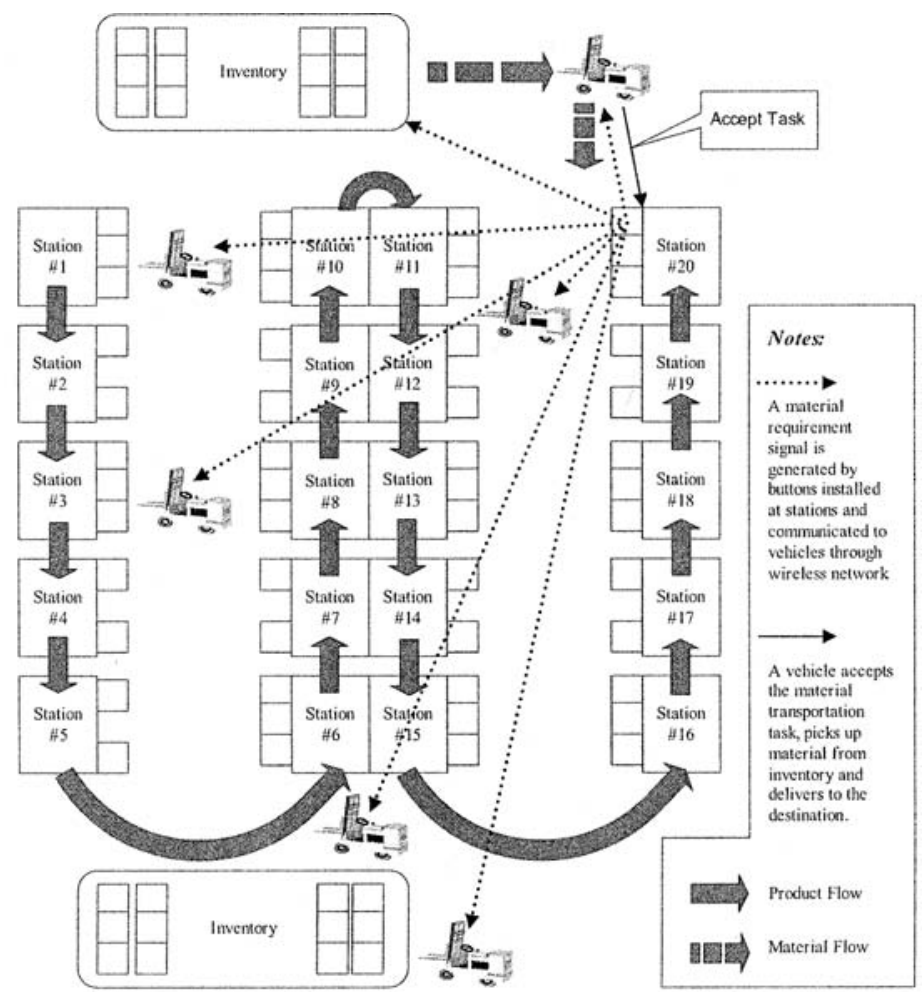

Figure 1 - A scenario of material handling simulation in an assembly line 
The following assumptions are clarified:

- Production type: mass production of mixed product models.

- Production organization: U-shaped production/processing line, which integrates the manufacturing processes into a balanced and continuous material flow.

- Modular/station arrangement: the flow-of-products oriented production layout asks for separation of the whole process to manufacturing stations according to the optimized process rate and the granularity of material control.

- JIT production control: a pull control mechanism is applied for the control of production. Products are carefully sequenced before going on the line. There is no WIP buffer arrangement on the main assembly line. Production rate is a constant that is optimized by operation research practices.

- There are many transportation vehicles (illustrated by the icon of a forklift) moving around the whole plant mounted with wireless communication capacities and simple transaction systems for material handling.

The JIT-based material handling approach we proposed borrows similar principles from JIT-based production control and JIT-based inventory management in that: the right material is delivered from its inventory to the right production site, at the right time and in the right amount. Here, material transportation in the plant floor is considered as individual tasks. A task requires a material Kanban (MKanban) to be delivered. In figure 1, a material request signal is firstly generated by an assembly station running out of a part supply; after occupying a material Kanban, the request is broadcasted to a number of vehicles moving in the scope of a wireless network; then through negotiation, the task is confirmed by a vehicle and being delivered finally to the right station.

In our view, JIT material handling based on Kanban concept is not merely a pure event based system. In an event system, an event calls for a system response immediately; while in a Kanban based system, a generated event gets processed only after obtaining a physical object - M-Kanban. In other words, the processing (transportation) of a material requirement event holds until the system releases a MKanban and the event is qualified to occupy this free Kanban among all other events. Based on a Kanban control mechanism, we believe that the material handling system is able to reach a natural balancing of material requirements and transportation activities through delicate arrangement and management of Kanbans.

In addition to the standard function in a simulation environment, such as discrete event generation, simulation clock generation and an animation interface, this system should have special functional modules that try to model and simulate the dynamics in the plant floor. Three groups of functions make up the JIT material handling system: scenario generation, simulator, and graphical user interface. Scenario generation maintains a large variety of configuration information relating to: 1) static scenario, such as plant layout, moving tracks, and a central part inventory; 2) changeable simulation parameter, such as number of vehicles and number of Kanbans. Simulator is the core of the software in that it controls the simulation of both the production and material handling processes. Each transportation vehicle has a separate vehicle simulation module to make its own decisions, including task sequence, task schedule, moving control, loading and 
unloading operations, or even collision resolution decisions if necessary. Station simulation is a module to simulate a simplified production process taking into consideration only the consumption and replenishment activities of materials/parts at each station. Kanban Simulation manages buffering and circulation (life-cycle) of M-Kanbans. It makes two kinds of decisions 1) allocation of material requirement signals to empty M-Kanbans; 2) allocation of M-Kanbans to vehicles. In the simulator, basic simulation facilities such as timer and random number generator should be provided to simulate synchronize events or discrete events. Graphical User interfaces are supposed to timely update the graphical simulation, system event, exceptions, etc, and provide timely response upon users' requests for any kind of simulation and statistical data.

A distinctive feature of the designated JIT material handling simulation is "quasi-realism". The proposed simulation system possesses functions that surpass traditional simulations. The most distinguishing one is its ability to facilitate runtime reconfiguration. For example, the arrangement of assembly tasks to manufacturing stations could be adjusted during the execution of a simulation, so that the bottleneck (of the line) and system responses could be constantly changing. Another example is that each component is manageable not only in its configuration parameter, but also controllable in its behaviors individually (for example, each vehicle is able to make schedules, control status, and choose its own delivery route). In contrast, other simulation systems read a batch file before each simulation launch. It is difficult for people to analyze dynamic system behaviors by changing system configurations in separate simulation launches.

\section{THE AGENT-BASED JIT MATERIAL HANDLING MODEL}

We use agent technology to model major components in the JIT material handling simulation system. Agents are sophisticated computer programs that act autonomously on behalf of their users, collaborate across open and distributed environments, to solve a growing number of complex problems. There are four kinds of agents designed in the simulator.

- Main Control Agent (MCA)

Main Control Agent (MCA) is responsible for simulation initialization, simulation termination, agent (thread) management, and thread synchronization. MCA also includes a timer and an event generator along with its main thread.

- Station Agent (SA)

Station Agent (SA) is a running thread simulating material requirement activities at stations. It is dynamically generated and destroyed by the MCA. A simple production rate of the assembly line is set for all stations to consume their required parts in certain amounts. So, with the progress of one production step, the material balances at stations may reach the requirement levels or the urgent levels. In extreme occasion, materials may be exhausted which causes the whole assembly line to stop.

- Kanban Schedule Agent (KSA)

Kanban Scheduling Agent (KSA) is a separate thread whose role is to take care of 1) the scheduling of material requirements to M-Kanbans, and 2) the assignment of M-Kanbans to vehicles. KSA is dynamically created and 
destroyed by the MCA. It applies regular and emergent scheduling strategies. Regular scheduling is fulfilled by the negotiation carried out between KSA and participating VAs.

- Vehicle Agents (VA)

Each Kanban assigned to a vehicle is confirmed by its VA and served by the VA through a series of actions. A vehicle agent is able to handle its local schedule, maintain its status, and controls its movement, repair, and resume actions. The threads for all vehicles are generated or destroyed by the MCA at the same time.

The four agents collaborate with each other and their interactive behaviors constitute the functionalities of the simulator. The MCA, located at the centre, functions as a core role for synchronizing. It generates two timely synchronizing events both for production and material handling process simulation. The simulation event is a regularly generated time event for pushing all simulation processes forward by one step. The frequency of simulation events is related to a specified speed set in the real-time mode for a simulation launch (or sometimes called an experiment). The production event is a regularly generated time event for pushing the production of products forward by one step. The time period for production event is set by the production rate of the assembly line. Two other Kanban events (Free Kanban Event and Kanban Reschedule Event) are used in material handling simulation and they will be further clarified in Section 4.2.

\subsection{Production Simulation}

By introducing a production event, a simple push mechanism with a constant production rate could be demonstrated. However, the realization of a pull mechanism needs much more effort. Figure 2 shows the principles of this pull control.

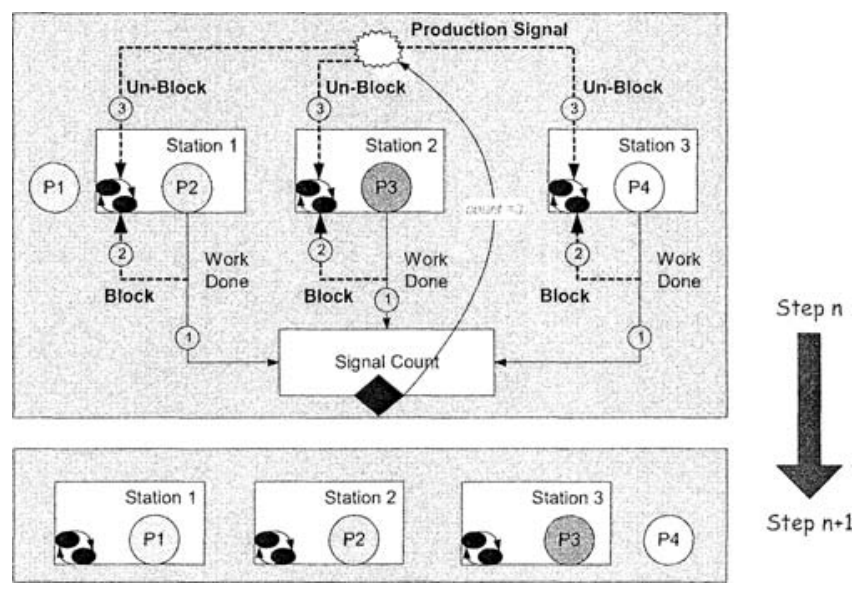

Figure 2 - Pull control of production simulation 
Each station is doing some operations to the product on hand, say, P2, P3 and P4. Assume that the time required for the operations at a station is not a fixed number due to 1) complexity of operations on different products; 2) experience and tiredness of human operators; 3 ) availability of tools, etc. In this occasion, a flexible production rate is required so that when and only when all stations finish their duties, the production is "pulled" forward by one step. In the next step, station 1 will grab a new product $\mathrm{P} 1$, and $\mathrm{P} 2, \mathrm{P} 3$, and $\mathrm{P} 4$ will switch to succeeding stations, respectively.

In Figure 2, once a station finished its operations on the holding product, it sends a Work Done signal to a counter and then blocks its status. Until the count of signals reaches the total number of stations (for example, the count should be 3 in Figure 2), a Production Signal is generated which then unlocks all stations from blocking status. In this occasion, all stations "pull" a product from their previous stations when a production signal unblock their status from "blocking" to "normal".

This simple pull mechanism creates a dynamic production line that could predict system performance under various "what-if" scenarios, such as bottleneck shift, change of task/schedule, or even task re-arrangement. However, from a practical view, an assembly needs a constant production speed since physical equipment serving the line is not dynamically adjustable. The operations at stations need to be measured and balanced carefully using operation researches. Having this in mind, this is what simulation software targeted for - the purpose of simulation is to simulate the "what-if" situations, rather than a playback of real production settings.

Both push and pull control strategies will be simulated for a sample layout illustrated in Figure 1. A station agent is used to simulate the production process and generates material requests in line with the production progress. At each production step, a station will hold a product for a time period that is defined for the tasks assigned to it. The materials/parts used by these tasks are consumed by certain amounts set by these tasks.

\subsection{Material Handling Simulation}

Material transportation and delivery is the primary purpose of this simulation system. Material handling simulation could only be achieved based on the availability of production simulation, since the source of material transportation tasks is the material requirements accompanied with the production process. A material handling event is generated by the station agents. There are two levels of material handling events to represents two situations happened at stations: Material Requirement (MR) and Material Query (MQ). A material requirement is an event signaling a situation when the stock level of the referred material/part is low at a station and a full package of this material needs to be delivered to this station. A Material query is an event designed to signal a higher level of material shortage at a station. After a MR signal, the station continues to consume materials/parts at each production step. At a certain level, a MQ event could be generated and indicate that the material/part requested in the previous MR event has not been delivered to the station yet. The current balance reaches such an emergent level that the station staff and material management personnel need to know immediately the process status of this material. The system automatically tracks the queried material on behalf of the users and reports the status of material delivery. 
In a pure event system, the generated MR or MQ are put into separate queues, and if possible, being processed immediately. In this system, we designed a material Kanban control mechanism on top of the event level. For the convenience of description, material Kanban is referred simply as Kanban in the rest of the paper.

Through correct choice of Kanban parameters and correct allocation of Kanbans in the layout of the production line, the material handling activities could reach natural balancing and optimization. A full material handling process can be divided into several steps (Figure 3):

1. Material requirement event, say MR1, is generated by a station and put in the MR event queue;

2. The MCA checks the Kanban Buffer regularly in each simulation circle and it identifies that an empty Kanban (designated by blue color) is available, say $\mathrm{K} 1$;

3. MCA generates a Free Kanban Event;

4. The KSA is triggered by the Free Kanban Event and picks MRI from the event queue so that it could occupy $\mathrm{K} 1$. The status of $\mathrm{K} 1$ now changes to "ready-to-schedule" (designated by yellow color);

5. The KSA scans the Kanban Buffer and it finds out that $\mathrm{K} 1$ is a Kanban that has not been assigned to any vehicle;

6. KSA negotiates with all VAs and select one vehicle, say V1, to deliver K1. The status of K1 now changes to "assigned" (designated by red color);

7. V1 put $\mathrm{K} 1$ in its schedule;

8. V1 deliver the required material from the part inventory to destination station;

a. V1 decided to withdrawn the task $\mathrm{K} 1$ from its schedule. This occasion is called "Kanban Re-schedule".

9. $\mathrm{K} 1$ is released by V1 and becomes an empty Kanban in the Kanban buffer;

10. V1 returns to the inventory site and picks the next Kanban from its schedule or becomes idle if there is no more task in its schedule.

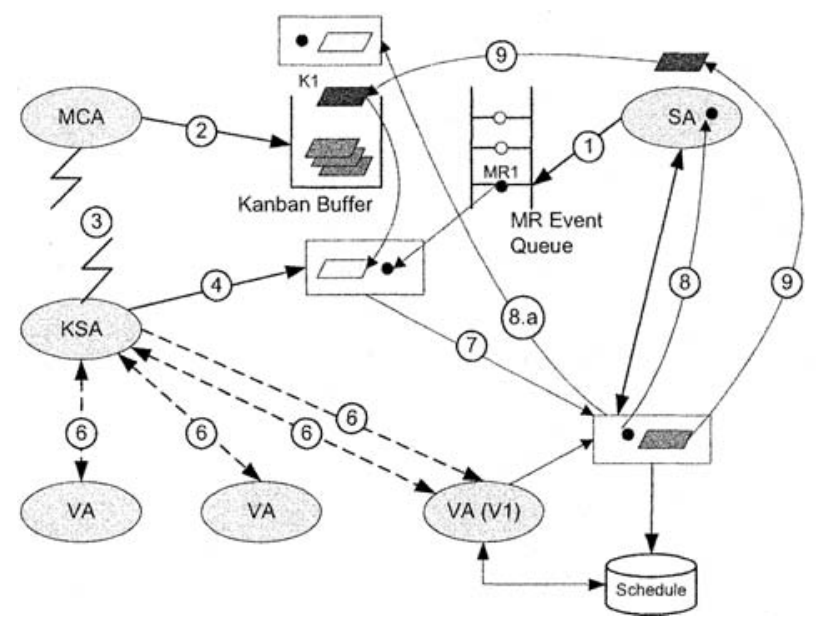

Figure 3 - Illustration diagram of Kanban handling 
In above process, the Kanban buffer is a kind of data storage for all Kanbans currently circulating in the simulation environment. It has several "containers" (logical structure) to maintain Kanbans with different properties. The term container is used to analogize its physical appearance in Toyota plant in 1970's, when Kanbans are cardboard cards circulating in small areas and being collected using containers. The number of Kanbans in each container represents the Kanbans that are allowed to circulate in a certain area in the production line, generally an aisle. Of course, Kanbans and their circulation are implemented through electronic means. They are processed by a data processing system which dynamically changes the information and status they carried. A Kanban could be found in a number of states, including "free", "ready-to-schedule", "bidding", and "assigned".

Two major issues are involved in the material handling process: Kanban scheduling and vehicle control, which are realized by two kinds of agents respectively. A bidding process similar to the Contract Net (Smith, 1980) is adopted to do Kanban scheduling between the KSA and multiple VAs. So, bidding processes are designed both in KSA and in VA. The control of vehicle is a multi-facet mission including: 1) motion control- moving, loading/unloading, stop; 2) status control -idle, active, repair, pause, load/unload, delivery, empty load; 3) route control - route plan, route choice, return route, collision resolution; 4) bidding process control giving a bid, responding to an awarded Kanban; 5) schedule control - local schedule, drop tasks, rearrange tasks; 6) Kanban status control - free, reschedule, delivery.

\subsection{Implementation}

A software prototype of the proposed JIT material handling simulation is implemented using a commercialized simulation tool - Anylogic ${ }^{\mathrm{TM}}$ of $\mathrm{XJ}$ Technologies ${ }^{\mathrm{TM}}$ (URL:http://Www.xjtek.com/anylogic/). Among others, one important feature of AnyLogic ${ }^{\mathrm{TM}}$ is its agent-modeling capability. Even though this feature cannot fully meet our agent-based simulation needs, it helps quick deployments of complex simulation models in a professional way. Because of its open architecture and pure Java implementation, it will not be difficult for us to integrate AnyLogic ${ }^{\mathrm{TM}}$ with the multi-agent framework AADE (Hao et al., 2005) to create a more powerful agent simulation environment.

Figure 4 shows a snapshot of the prototype environment. A full working prototype will be available for demonstration at the conference. 


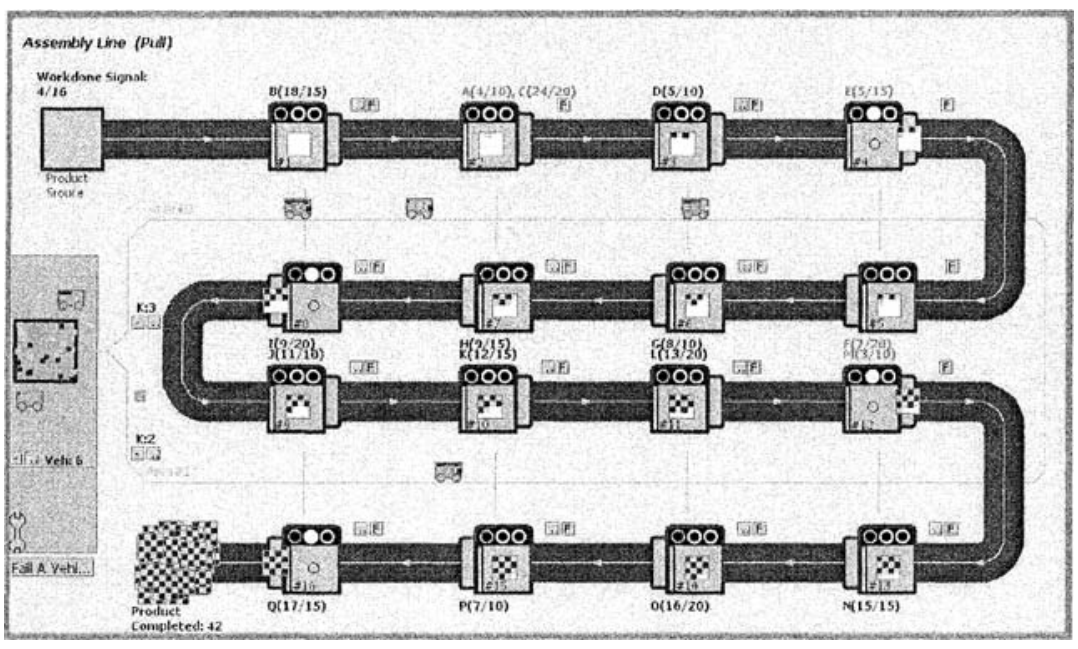

Figure 4 - A snapshot of the simulation prototype

\section{CONCLUSION}

Based on requirements of industrial partners, material handling has been recognized as a loose loop in most assembly plants. Just-in-time is a pervasive paradigm implemented in nowadays manufacturing plants that strive to survive in a global competition. The principles of JIT bring forward an optimized production environment and a mechanism for waste less inventory replenishment. However, material handling problem, especially the material/part supply at the plant floor level, is seldom addressed in research literature and in practices. In this paper, we propose a material handling simulation system that applies JIT principles. Material Kanban is an entity that carries a material request and represents a material transportation task. An agent-based simulation environment is designed and a prototype system is implemented using AnyLogic ${ }^{\mathrm{TM}}$. Many experiments will be performed based on the simulation model build for this purpose.

The JIT-Based material handling is expected to bring forward a number of advantages, such as optimization of stocks levels at production stations / cells, balancing of transportation load in the whole plant floor, obtaining manageability on material handling performance and accurate prediction and optimization of transportation resources. The major difference of this simulation from others is that the flexibility of the agent-based approach facilitates the simulation of various "what-if" scenarios including different layout designs, objective parameters and dynamic situations in the plant floor. 


\section{REFERENCES}

1. Askin, RG. Manual and operator-based transfer batch sizes in JIT manufacturing. In Proceedings of Promat'99, Chicago, IL, pp.1-21, 1999. Reprinted in Progress in Material Handling Practice: 1999, Material Handling Institute, Charlotte, NC, pp. 167-87.

2. Berkley, BJ. A review of Kanban production control research literature, Production and Operations Management 1992;1(4): 393-411.

3. Gupta, SM and Al-Turki, YAY. The effect of sudden material handling system breakdown on the performance of a JIT system. International Journal of Production Research 1998; 36(7): 1935-60.

4. Hao, Q, Shen, W, Zhang, Z, An Autonomous Agent Development Environment for Engineering Applications, International Journal of Advanced Engineering Informatics, 2005, 19(2):123-134.

5. Hopp, W and Spearman, M. Factory Physics: Foundations of Factory Management. Irwin/McGraw Hill, Chicago, IL, 1996.

6. Krishnamurthy, A, Suri, R and Vernon, M. Re-examining the performance of MRP and Kanban material control strategies for multi-product flexible manufacturing systems. International Journal of Flexible Manufacturing Systems 2004; 16(2):123-50.

7. Kusiak Andrew. Computational Intelligence in Design and Manufacturing. John-Wiley \& Sons, Inc., 2000.

8. Martins, $\mathrm{K}$ and Lewandrowski, U. Inventory safety stocks of kanban control systems. Production Planning and Control 1999; 10(6):520-29.

9. Shen, W, Norrie, DH and Barthes, JP. Multi-Agent Systems for Concurrent Intelligent Design and Manufacturing. Taylor and Francis, London, UK, 2001.

10. Shirk, WT. Material flow controls in a JIT environment. In Proceedings of the Technical Program: National Electronic Packing and Production Conference (NEPCON EAST'88), pp.325-332, 1988.

11. Smith, RG. The Contract Net Protocol: high-level communication and control in a distributed problem solver. IEEE Transactions on System, Man, and Cybernetics 1980; C-29(12): 1104-13.

12. Uzsoy, R, Martin-Vega, LA. Modelling Kanban-based demand-pull systems: a survey and critique. Manufacturing Review 1990; 3(3):155-60.

13. Venkataramanaiah, S, Krishnaiah, $K$ and Pichandi, R. Management of WIP inventory in JIT environment under cellular layout - a case study. Journal of Scientific \& Industrial Research 2001; $60(3): 256-63$.

14. Womack, JP and Jones, DT. Lean Thinking: Banish Waste and Create Wealth in Your Corporation. Simon and Schuster, New York, NY, 1996. 